\title{
El reconocimiento de la actividad investigadora universitaria como mecanismo de regulación del mercado académico*
}

\section{Recognition of university research activity as a mechanism for regulating the academic market}

José Luis San Fabián Maroto ${ }^{1}$

Recibido: 20 de noviembre de 2019 Aceptado: 12 de diciembre de 2019 Publicado: 31 de enero de 2020

To cite this article: San Fabián, J. L. (2020). El reconocimiento de la actividad investigadora universitaria como mecanismo de regulación del mercado académico. Márgenes, Revista de Educación de la Universidad de Málaga, 1 (1), 23-44

DOI: http://dx.doi.org/10.24310/mgnmar.v111.7208

${ }^{1}$ José Luis San Fabián Maroto 0000-0002-8149-7797

Universidad de Oviedo

jlsanfa@uniovi.es

“Nada vale la ciencia si no se convierte en conciencia" (Carlo Dossi, 1849-1910)

\section{RESUMEN}

Durante los últimos años se han implantado en las universidades de forma generalizada políticas de evaluación y reconocimiento de la actividad investigadora, cuyos fundamentos y efectos son objeto de revisión en este artículo. La producción científica se ha convertido en un criterio clave para la promoción individual del profesorado, el prestigio y la calidad de las universidades y, en consecuencia, en una de sus principales fuentes de poder y legitimación. Ello ha incidido en las dinámicas institucionales, modificando las relaciones entre los profesionales y sus hábitos investigadores a través de lo que se ha denominado cultura del impacto. A partir de un análisis crítico que evidencia los efectos perversos de los sistemas de evaluación implementados, se reflexiona sobre los principales dilemas que plantean y se realiza un conjunto de propuestas dirigidas a combatir lo que puede considerarse como la reforma encubierta más profunda de la universidad en estos últimos años.

Palabras clave: universidad; reconocimiento de la actividad científica; evaluación de la calidad investigadora; cultura del impacto

\section{ABSTRACT}

During the last years, policies of evaluation and recognition of the research activity have been implemented in universities, whose foundations and effects are subject to revision in this article. The scientific production has become a key criterion for the individual promotion, the social prestige

* Este artículo deriva de una conferencia titulada El reconocimiento de la actividad investigadora universitaria ¿estímulo o freno? impartida por el autor en el 5을 Congreso Internacional de Investigación Educativa en la Universidad Autónoma de Hidalgo, Pachuca (México). 
and the quality of the universities, and consequently in one of its main sources of power and legitimation. This has influenced the institutional dynamics, modifying the relationships between professionals and their research habits through what has been called impact culture. From a critical analysis that shows the perverse effects of the implemented evaluation systems, the author reflects on the main dilemmas raised, in order to make a set of proposals aimed at acting against what can be considered the most relevant covert reform of the university in these years.

Keywords: university; recognition of scientific activity; evaluation of research quality; impact culture

\section{EL DEBATE SOBRE EL RECONOCIMIENTO, SUS POLÍTICAS Y EFECTOS}

La producción de conocimiento científico, esto es, la investigación, es una de las funciones básicas de la universidad, junto con la transmisión de ese conocimiento a través de la docencia. Las políticas de reconocimiento, a través de la evaluación de la producción científica, no solo se han convertido en el principal instrumento para el acceso, estabilización y promoción del profesorado universitario, sino que además configuran la propia profesión, condicionando las relaciones entre investigadores, su financiación y formación, las metodologías científicas, los procesos de difusión e impacto de los resultados, el acceso a cargos académicos y de gestión, la formación de doctores, etc.

En el ámbito de la filosofía existe un debate sobre el reconocimiento como una necesidad humana vital, donde su negación equivaldría a una forma de injusticia (Grueso, 2017). Muchos conflictos sociales son conflictos de reconocimiento y numerosos movimientos sociales surgen, en buena parte, por la falta de reconocimiento de una identidad colectiva (de clase, género, etnia, sexual...). La sociedad es un sistema al que los ciudadanos contribuyen con su esfuerzo esperando recibir a cambio servicios, derechos, garantías... y un reconocimiento de sus valores. Aquí nos referimos a un aspecto específico de este reconocimiento, el vinculado al desempeño laboral, a la identidad profesional si se prefiere, atravesada necesariamente por esas otras identidades colectivas.

El acceso y promoción en la carrera profesional universitaria se lleva a cabo a través de sistemas de reconocimiento de méritos de carácter racional-burocrático. Su finalidad explícita es asegurar la racionalidad del sistema en términos de calidad, igualdad y servicio a la sociedad. Sin embargo, su aplicación puede producir efectos no deseados pervirtiendo dicha racionalidad. Esto lleva a preguntarnos ¿cómo se define el mérito investigador?, ¿cómo es evaluado?, ¿cuáles son sus efectos?

Basándose en los dos modos de producir conocimiento definidos por Gibbons et al. (1997), Lya Sañudo (2017) señala que en el Modo 1, producido mayormente en la universidad y centros de investigación reconocidos, los posibles usuarios prácticos y agentes de decisión tienen poco acceso al conocimiento producido, ya que la difusión se realiza principalmente en medios especializados con una dinámica propia que es independiente de la demanda para resolver problemas. La distribución se realiza prioritariamente dentro y para el gremio especializado de la disciplina. En 
el Modo 2, la investigación no está institucionalizada, el conocimiento se produce en los lugares más diversos y es distribuido de acuerdo a los intereses de distintos agentes, cuya relación con los usuarios es intensa y estrecha, lo que permite una distribución de los resultados dirigida a las organizaciones sociales con las que interactúan. En esta segunda lógica, de acuerdo a Gibbons, et al. (1997), la innovación producida en los procesos de investigación posibilita la solución de problemas a partir del trabajo colaborativo entre científicos y usuarios. La creatividad se propicia dentro del grupo de investigación y los usuarios, asociada a la comunidad a la que está dirigida, sus condiciones, potencialidades y limitaciones; contribuyendo al empoderamiento de la comunidad, no a ampliar la brecha social.

\subsection{El estilo de producción indexada}

Dado que la producción indexada ha adquirido un rol destacado en la construcción de la identidad intelectual universitaria, necesitamos preguntarnos por las características de este estilo de producción. En la década de los 70, en los EE.UU. se empezaron a aplicar de forma generalizada sistemas de evaluación para la asignación de fondos, subvenciones e incentivos de investigación. Desde entonces, los gobiernos y las universidades de numerosos países han ido implantando diversos sistemas de evaluación de la producción investigadora, creando organismos y agencias de evaluación y acreditación, e incorporando nuevos procedimientos y herramientas (revisión por pares, indicadores de impacto, índices bibliométricos, etc.).

A mediados del siglo pasado, el Dr. Eugene Garfield fundó el Institute for Scientific Information (ISI), que posteriormente pasó a manos de la empresa Thomson Reuters Science, una división de Thomson Reuters Corporation autodefinida como "la fuente líder en el mundo en información inteligente para investigadores" (Herrán y Villena, 2016). Su producto estrella, la Web of Science (WoS), provee cobertura a las principales revistas de mayor influencia en todo el mundo. En 1955 Garfield revolucionó la investigación científica con el concepto de indexación de citas e investigaciones (Journal of Citation Report, JCR), donde el factor de impacto de una revista es el cociente entre el número de citas recibidas de artículos publicados en varios años anteriores y el número total de artículos publicados por la revista en esos años ${ }^{1}$. Las revistas que están incluidas en la WoS corresponden a las que años anteriores estaban en el listado del ISI.

Un problema es que la ISI era altamente selectiva y restrictiva, en cuanto a idioma, dominio geográfico, campo disciplinar, etc., de forma que la producción científica de muchos países, mayormente no anglosajones, y de algunas áreas, como las de Ciencias Sociales, quedaban infrarrepresentadas. En 1997 se genera un proyecto piloto para la publicación electrónica cooperativa de revistas científicas en Internet, el Scientific Electronic Library Online (SciELO), especialmente desarrollado para

1. La Web of Science es un índice académico de citaciones en línea provisto hasta hace poco por la empresa Thomson Reuters a través de su plataforma Web of Knowledge. Actualmente la plataforma WoS ha sido adquirida por la empresa Clarivate Analytics (Onex Corporation y Baring Private Equity Asia). 


\section{EST UDIOS Y ENSAYOS}

responder a las necesidades de comunicación científica en los países en desarrollo y particularmente de América Latina y el Caribe, como una solución para facilitar la visibilidad y el acceso universal a su literatura científica, contribuyendo a la superación del fenómeno conocido como 'ciencia perdida'. Aun así, la presencia iberoamericana en ESCI es secundaria. Los investigadores del primer mundo apenas citan la producción latinoamericana o de países no anglosajones, porque no aparecen en los medios que ellos toman en consideración ni en los sistemas de indexación internacionales (Gibert-Galassi, 2013). Según este sociólogo chileno, las instituciones y practicantes de las ciencias sociales han sido históricamente dependientes de sus contrapartes en los Estados Unidos, Francia, Inglaterra... en términos de ideas, conceptos, teorías y métodos, en un estado del arte caracterizado como dependencia académica del sur respecto al norte. Hoy existe una polarización entre dos estilos (ISI y SciELO) según países y disciplinas, y aunque la producción de las ciencias sociales latinoamericanas se ha incrementado de forma exponencial durante las últimas dos décadas, sus productos tienen aún baja visibilidad y ocupan un lugar subordinado en el mundo académico (Gibert-Galassi, 2015, pp. 30).

Las características básicas de la evaluación basada en la indexación -“isificación"-son:

a. Su carácter formal. El contenido de los artículos es considerado cada vez menos relevante, lo que se valora es la calidad del medio en que se publica, más que el producto mismo se tiene en cuenta el índice de impacto de la revista en la que se publica. Al aplicarse masivamente, la evaluación de la investigación tiende a degenerar en un proceso burocrático. Lo que se controla es el ajuste formal de las publicaciones a unos estándares dependientes del prestigio del medio publicado, no del producto en sí. Si el medio o canal de difusión es de calidad contrastada, se considera que no es necesario juzgar de nuevo el contenido; de forma que puede haber artículos valiosos que se desechan porque se han publicado en revistas de reducida difusión, y viceversa, por qué no.

b. Se trata de una evaluación subsidiaria. No se evalúan informes de investigación, solo publicaciones. Por lo general, la atención se centra en el número de "éxitos" que los investigadores pueden mostrar, como el "factor de impacto" y otras medidas de rendimiento que las revistas aplican para dar cuenta de su valor y utilidad.

c. Emplea de forma sistemática la evaluación por pares, "peer review", en su doble vertiente, ex ante (proyectos de investigación, becas, contratos) y ex post (sexenios, acreditación, proyectos de investigación, programas, instituciones), siguiendo criterios fijados por los comités editoriales de las revistas. En realidad, quienes evalúan son las revistas y sus revisores. "Las revistas académicas de punta forman parte de sistemas que definen el conocimiento al aprobarlo en nombre de los expertos que revisan o editan lo que se publica. A través de las revistas académicas, los descubrimientos científicos son "autorizados" en el sentido weberiano de autoridad" (Post et al. 2013, pp. 4). 


\section{EST UDIOS Y ENSAYOS}

d. Se impone una modalidad de formato, el artículo, excluyéndose en la práctica el resto, como libros y monografías, que han supuesto hasta ahora un $40 \%$ de las publicaciones en CC.SS.

El uso de factores de impacto y rankings para evaluar publicaciones, instituciones e investigadores es relacionado por Post et al. (2013), editores de la revista Comparative Education, con cuatro movimientos:

- La racionalización del conocimiento experto, asumido como una característica inherente a la autoridad burocrática.

- La política de regulación y control de la educación superior, que se manifiesta en el nuevo "gerencialismo" y sus intentos de evaluar la investigación.

- Las políticas de precios y finanzas de las editoriales académicas comerciales, que han dejado de ser financiadas por suscriptores individuales.

- Las expectativas de generar una actuación cada vez más dramática por parte de los editores de revistas académicas ("cosmetólogos"), aun cuando estos se vean a sí mismos más como anfitriones de la convivencia y del pensamiento que como empleados de línea en una fábrica de currículos.

Gibert-Galassi (2015, pp. 25-26), tras realizar un análisis de la producción indexada en CC.SS. en cinco países -Argentina, Brasil, Chile, Colombia y México-, concluye que la tensión entre las múltiples tareas que deben enfrentar los académicos, el equilibrio entre la vida personal y la vida laboral, y la sobrecarga laboral contribuyen a la "precariedad de las identidades académicas", generando fragilidades en el ámbito:

a) Ontológico: la fragilidad de "ser" académico, tensionado entre la angustia académica de producir para obtener fondos y el estar permanentemente compitiendo y exponiendo en congresos y otras actividades.

b) Cuasi-legal: en el régimen neoliberal la disminución del financiamiento público para investigación ha hecho posible el recorte de las nóminas y la contratación o recontratación de muchos académicos que ayer tenían puestos estables en puestos esporádicos o de tiempo parcial.

c) Reputacional: la fragilidad del estatus del académico y de la propia universidad, que en función de los rankings pueden caer en el escalafón, perder reputación y, con ello, las matrículas o las donaciones, generándose un círculo de retroalimentación con las fragilidades citadas.

Para este autor, el fenómeno mundial de la indexación de las publicaciones científicas se vincula a la racionalidad capitalista de la ciencia, lo que se manifiesta en "la precarización del empleo, la orientación productivista del enfoque neoliberal y el rol del dinero en algunas disciplinas, ya sea por el lado de los productos (como la biotecnología) o los servicios (como la economía y la ciencia política)" (op.cit, pp. 26). 
La irrupción en la academia de las publicaciones en acceso abierto (Open Access) presumía que iba a romper el monopolio de las grandes empresas editoriales y a transformar el modo imperante de difusión basado en revistas indexadas fundamentalmente entre colegas y expertos (modo 1), al abrir el acceso del conocimiento a través de bases de datos, revistas electrónicas, blogs, que son puestos al día constantemente y consultables por todo el mundo (modo 2). Sin embargo, el acceso abierto no ha modificado de forma sustancial la distancia entre los teóricos y los prácticos: sigue siendo un acceso restrictivo a grupos que carecen de in/formación necesaria para su selección y utilización. Como indican Post et al. (2013), varias revistas electrónicas, que empezaron de libre acceso, ahora cobran a las bibliotecas cientos de dólares. Se da la paradoja de que se admite el valor de la publicación en abierto para difundir la investigación, pero no siempre se reconoce su valor científico en los procesos de evaluación, excepto si es una revista OA incluida en WoS (Giménez Toledo, 2014). Mantener como referencia casi obligada las bases de datos de WoS o Scopus para la valoración positiva de una contribución científica restringe de facto las posibilidades de que los investigadores decidan publicar en revistas de acceso abierto.

La evaluación de la investigación constituye el principal instrumento para estabilizarse y promocionar en la academia, donde WoS (Web of Science) y Scopus (Elsevier) son el "epicentro" de un sistema sobre el que giran otros subsistemas como el político (las universidades), el productivo (campos y áreas científicas) y el gestor (las revistas y las agencias de evaluación). El JCR se ha convertido, en la práctica, en un sistema único y excluyente para el reconocimiento de trabajos, personas, departamentos, centros universitarios y de las propias revistas (Herrán, 2012; Herrán y Villena, 2016). Este sistema de evaluación de la productividad científica está cambiando los hábitos del profesorado universitario. Los investigadores, en lugar de tener como objetivo de su actividad científica descubrir nuevos conocimientos, han de orientarla necesariamente a obtener el mayor número de publicaciones consideradas como valiosas por dichos indicadores (Torres López, 2013). Refiriéndose a México, Rincón Ramírez (2017, pp. 86) afirma que la imposición de las políticas públicas destinadas a la evaluación de los académicos forzó la producción del conocimiento, más por la necesidad de no ser excluidos y marginados de las "bondades" que otorgan los diversos programas de estímulos que por la importancia de abordar problemas concretos de la realidad social. No importa mucho lo que se produce ni para qué se produce, sino producir para estar dentro de los sistemas de estímulos. ¿Cuáles son los efectos en las "dinámicas institucionales" de este modo de producción?

\subsection{Incidencia del sistema de impacto en las dinámicas académicas universitarias}

Los rankings de revistas, a través de los esquemas de medición del desempeño, contribuyen a la prevalencia de la performatividad en la vida y los procesos de investigación de muchos académicos. A partir de la literatura sobre la identidad, Gendron (2008) relata la construcción del actor académico -academic perfomer- 
a través de los rankings de revistas, en términos de actitudes y comportamiento, sostenido mediante prácticas de medición del desempeño que influyen significativamente en la forma en que se concibe y se lleva a cabo la investigación, ejercitando una influencia restrictiva en la libertad académica. Los científicos, como intérpretes o ejecutantes, están fundamentalmente preocupados por establecer y mantener su reputación, y esta búsqueda de reputación genera tensiones entre la innovación y la conformidad. La productividad "publicacional" no tiene mucho que ver con el objetivo de la actividad científica, descubrir nuevos conocimientos, y, paradójicamente, el énfasis excesivo en la medición del desempeño y en el ranking de las revistas puede traducirse en un estancamiento intelectual. Según este autor (op.cit, pp. 19), "la generación perpetua de la incertidumbre en las ciencias es supervisada y controlada a través de este sistema formal que asegura que la investigación nunca llega a ser demasiado 'original'. La innovación y la novedad se atenúan así siempre por las exigencias del sistema de control".

Tras una amplia revisión de la literatura sobre la impronta del paradigma dominante de evaluación en este ámbito, Manzano-Arrondo (2017) señala la debilidad de los supuestos en los que se asienta el uso de la TRC (tasa restringida de citación) y de los rankings; las consecuencias indeseables en términos de adaptación estética e incluso de fraude en torno a la producción científica; el deterioro de los principios rectores que se le suponen a la ciencia; un profundo desequilibrio de trato entre regiones, idiomas, disciplinas y enfoques, y efectos psicológicos y laborales negativos derivados de la instrumentalización del personal universitario. Verónica Walter (2017, pp. 16), al analizar los sistemas de evaluación del trabajo universitario en Argentina y España, concluye que "las políticas de evaluación constituyen dispositivos hegemónicos de regulación del campo universitario que operan como instrumento racionalizador de recursos; herramienta de asignación de posiciones, clasificación y jerarquización de los actores en el espacio universitario y dispositivo generador de prácticas académicas". Esta autora advierte que los procesos de evaluación propician formas de organización del trabajo académico caracterizadas por el individualismo, las acciones estratégicas, el simulacro, la competencia, la burocratización y el estrés, concluyendo que la evaluación constituye un instrumento de subjetivación que configura particulares formas de habitar la universidad. Desde un enfoque de origen foucaultiano, Saura y Bolívar (2019) describen la construcción del "sujeto académico neoliberal" y las nuevas subjetividades académicas vinculadas a los actuales modos de gobernanza digital que utilizan un "régimen de cuantificación" para construir "sujetos académicos bibliometrificados y digitalizados".

De manera más específica, destacamos a continuación los principales efectos del modo de producción indexado en el trabajo universitario:

a) Las prácticas de escritura y publicación académicas se ven inscritas en las reglas de un juego de saber-poder que descalifica ciertas formas discursivas y privilegia otras, con el objetivo de uniformar la producción académica y de ajustar las publicaciones a estándares internacionales; un régimen de verdad sobre aquello 
que puede o no llamarse escritura académica (Ortiz, 2013). Se impone un estilo narrativo minimalista -"esquematismo científico"-, con un número de páginas y una estructura establecida por los libros de estilo de los consejos editoriales. Este estilo, consistente en subdividir una investigación hasta la mínima unidad publicable, limita la capacidad de reflexión crítica y el análisis en profundidad de los temas. La presión para publicar en determinadas revistas como condición para promocionar conduce a la obsesión por publicar prematuramente, a la publicación compulsiva: escribir lo que sea con tal de que se publique. "La periodicidad y cantidad de los programas de evaluación desalienta los trabajos de investigación de largo plazo, que frecuentemente son desplazados por proyectos de corto alcance" (Buendía et al., 2017, pp. 982).

b) Las revistas internacionales de impacto promueven el dominio de determinados paradigmas de investigación y dificultan la difusión de paradigmas heterodoxos. Las revistas científicas se convierten en algo intocable, indiscutible, en vez de ser foros de interlocución y debate académico. Se ejerce una propagación de los modelos de investigación positivistas desde el campo de las Ciencias Naturales al de las Ciencias Sociales, en sus aspectos formales y metodológicos, generando una estandarización marcada por la impostura de las Ciencias Técnicas (ingenierías, biología, etc.), que imponen su metodología y formato de producción como hegemónicos para el conjunto de las ciencias. Las agencias de evaluación actúan como tribunales inquisitoriales de metodología que institucionalizan una forma o versión de la ciencia (Cheek, 2013).

c) Otra consecuencia de esta noción de impacto es la "cultura de la citación" (Wouters, 1999). La concepción de la cita como moneda de pago (reconocimiento del valor del trabajo de un autor consultado para la investigación), como proceso cognitivo, como acto persuasivo o como concepto simbólico, alimenta numerosos debates y estudios que han tratado de identificar las motivaciones para la citación, validar la relevancia de las citas o demostrar la inutilidad de las citas como medidas de calidad (Arencibia y de Moya Anegón, 2008). Se hace manipulación para alterar el índice de citación. Surgen los círculos de citas ("yo te cito, tú me citas"), revistas que remiten las referencias de sus artículos publicados a investigadores que trabajan temas afines con la finalidad de incrementar sus índices de citación. "Hay editores que están haciendo auténtica 'ingeniería del índice de impacto', de manera que recomiendan o incluso exigen a los autores citar artículos publicados en su revista (más en concreto, de los dos últimos años), si es que quieren publicar en ella" (Palomero Pescador, 2010, pp. 1).

d) Existe una contradicción entre la tendencia de la investigación a conformar equipos y los programas de estímulos orientados individualmente; se predica colaboración pero se practica competición, lo que enrarece las relaciones profesionales. "Desde un punto de vista colectivo, las dinámicas hegemónicas contribuyen a la fragmentación del cuerpo docente, aislando a los profesores de modo que se dedique 'cada cual a lo suyo', atentando contra lo común del trabajo y dejando a los docentes 'a 
la deriva', expuestos por separado, en solitario a las exigencias de productividad" (Walter, 2017, pp. 17). En referencia a las carreras de sociología de las universidades públicas en Bolivia, Torrez (2013, pp. 109) describe el escenario como campos de lucha política donde predominan intereses de cuño corporativo, sectoriales o de grupo, subordinando la propia gestión académica: "Las carreras de sociología y sus diferentes tribus universitarias operan en campos de disputa política donde se concentra el poder y se acumulan los conflictos en torno a intereses eminentemente corporativos/grupales", y no como comunidades científicas dedicadas al debate, la investigación y la reflexión sobre la ciencia. La propia autoría colectiva puede verse pervertida por la estructura de poder universitario, apareciendo profesores o gestores como autores de artículos que se publican en su departamento o grupo, aunque su implicación real en dichos trabajos sea mínima.

e) Se promueve una desvaloración y precarización de la docencia respecto a la investigación. El peso que se otorga a los méritos de investigación en forma de publicaciones en revistas es desmedido, cuando la mayoría de aspirantes a la universidad son contratados por necesidades docentes. La interacción entre la dedicación docente y la dedicación investigadora, en vez de adquirir un carácter complementario, genera tensiones entre ambas funciones, acentuadas con la aplicación de un sistema de incentivos a la investigación desequilibrado, que actúa en detrimento de la docencia, de forma que se asigna mayor "carga docente" a quienes muestran menos méritos investigadores, generando un círculo vicioso: al que más tiene más se le da, con el consiguiente deterioro de la atención a la docencia y al estudiante.

f) La investigación se aleja cada vez más de la práctica y de los problemas de los prácticos. Mientras que los grupos de profesionales centran más su investigación en los contextos de aplicación y a difundirla en sus redes formales e informales, los ámbitos universitarios tienden a hacer investigación dirigida a educación superior y a publicar preferentemente en revistas indexadas internacionales. Se impone una determinada concepción de "transferencia del conocimiento", que se identifica con la publicación en determinadas revistas académicas. Sin embargo, la transferencia e impacto de un artículo educativo puede ser mayor en una revista "profesional" de carácter nacional accesible al profesorado o responsables educativos que en una revista científica internacional en otro idioma consultada por académicos. Las dificultades para incorporar a los prácticos en la investigación universitaria contribuyen a su baja incidencia en la mejora de la práctica social (Sañudo, 2017). Hay una escasa valoración de la perspectiva local de la investigación, cuyo alcance más restringido suele ser considerado como un indicador negativo, cuando podría ser indicador de una relación entre la investigación y los problemas sociales. El resultado es un conocimiento con bajo sentido y compromiso social, carente de una agenda de reivindicación de las necesidades más sentidas de los sectores marginados, desprotegidos y excluidos (Rincón Ramírez, 2017).

g) El "pago por paper" se ha convertido en práctica generalizada (Opazo, 2017). Muchas revistas exigen pagos para revisar, traducir o publicar artículos, de for- 
ma que la capacidad investigadora se ve condicionada por el poder adquisitivo de los autores. A más publicaciones más recursos para investigar, creándose un círculo vicioso. Ello condiciona la identidad del profesional universitario, presionado por la necesidad de mostrar resultados y cuyo rol se encuentra en tránsito del docente-investigador al publicador-evaluador, dado que su prestigio y honorarios provienen de su visibilidad en las revistas para publicitarse y poder acceder a fuentes de financiación públicas y privadas. Para Julianne Cheek (2013), recibir financiación externa para llevar a cabo la investigación no es un acto neutral, los investigadores sufren cada vez más presión para asegurar la financiación y esta presión convierte la investigación en un bien que se compra y se vende. Por otra parte, el incremento del número de investigadores no se ve correspondido por el volumen de recursos, lo que conduce a una situación de embudo y genera desigualdad: "las repercusiones de los premios, 'estrellas' y 'medallitas' de los distintos programas son inequitativos y estratifican a los académicos, quienes compiten en desigualdad de condiciones para alcanzar los indicadores, debido a las diferencias en términos contractuales, en condiciones formativas y en apoyo institucional" (Buendía et al., 2017, pp. 982). Según estos autores, los efectos de la evaluación están contribuyendo a la precarización de los contratos y afectando al relevo generacional y la renovación de la planta académica.

h) Por otra parte, el sistema de evaluación indexada permite y fomenta la picaresca y las malas prácticas. Se incentiva el plagio y el corta y pega, la publicación por entregas para inflar el impacto, incluyendo aspectos parciales de un estudio, con la consiguiente reiteración y pérdida de sentido del conjunto, estilo salami de publicación (Moed, 2005), consistente en cortar y pegar partes de un trabajo ya publicado y obtener nuevas publicaciones para incrementar la productividad. Surge la figura del antropófago académico, del acaparador de títulos y certificaciones. Aparecen cientos de "editores depredadores"2. Se potencia el clientelismo, las redes de poder entre académicos -y políticos-, como muestran los casos de plagio y corrupción que aparecen periódicamente en la prensa y que afectan a diferentes universidades y países. Estas malas prácticas, resultado de "la 'patología organizacional' de la lucha por el factor de impacto como 'mercancía' rentable" (Santos Rego y Fernández-Ríos, 2016, pp. 1), son alimentadas por un sistema de "incentivos perverso" (Calver, Lilith y Dickman, 2013), configurando un "estilo de pensamiento profesional", un modelo de socialización profesional -"performer academic"- que dificulta la creatividad y limita la pluralidad científica (FernándezRiosa y Rodríguez-Díaz, 2014); un "fetiche" mal utilizado en comparaciones cienciométricas (Balaban, 2012, citado por Fernández-Riosa y Rodríguez-Díaz, 2014), y que ejerce una "atracción fatal" sobre los investigadores (Van Raan, 2005)33.

2. Ver: http://scholarlyoa.com/publishers/

3. Más de dos tercios de los artículos invalidados o retirados de la literatura científica son resultado de algún tipo de mala práctica científica, según un estudio publicado en PNAS: 43,4\% por fraude o sospecha de fraude; 14,2\% por publicación duplicada y 9,8\% por plagio (Fang, Grant Steenc y Casadevall, 2012). 
Todas las expresiones de la vida académica quedan afectadas por esta cultura del impacto. Los congresos y las reuniones científicas ofrecen como reclamo la publicación de los mejores papers enviados en revistas o editoriales de impacto, convirtiéndose en instrumentos para alimentar la carrera de méritos. Los programas de doctorado también se ven condicionados, las tesis pierden el carácter de investigación global y se fragmentan en artículos cuya selección final para formar parte de la tesis se delega en las revistas que, en función de su índice de impacto, deciden la estructura y plazos de defensa de las tesis doctorales. La difusión abierta de los resultados de las tesis se retrasa hasta que se haya asegurado su publicación. En las revistas "más cotizadas" los tiempos de espera para la revisión de los artículos pueden superar el año, lo que retrasa el acceso al conocimiento (Opazo, 2017).

Todo ello muestra cómo un modelo particular de producción, incentivación e intercambio científico se ha convertido en un instrumento para jerarquizar revistas, investigadores, centros y universidades, a través de lo que se ha denominado "la tiranía de los índices de impacto" (Palomero Pescador, 2010); donde la "cultura del impacto" determina la valoración del docente-investigador (Herrán y Villena, 2016), conformando un "estilo de pensamiento índice de impacto" (Fernández-Ríos y Rodríguez-Díaz, 2014). Ready (2014), refiriéndose a "la invasión del impacto" en el Reino Unido, afirma que la academia ha sido capturada por la agenda del impacto en la cual la utilidad de la investigación académica se juzga en contra de su utilidad social y económica más amplia.

\section{DILEMAS DE LAS POLÍTICAS DE RECONOCIMIENTO: DE LA EXCELENCIA A LA SUPERVIVENCIA}

\subsection{Calidad, Creatividad y Diversidad metodológica versus Cantidad y Uniformidad}

El modo de producción indexado pone el énfasis en la cantidad y en la homogeneidad, con una concepción acumulativa - bancaria- del conocimiento. La productividad se mide por la cantidad de trabajos publicados en determinadas revistas y la buena investigación es aquella de la que derivan muchas publicaciones. El sistema de evaluación establecido restringe y uniforma el conocimiento científico en su formato y contenido. Las revistas que ocupan los puestos superiores en los rankings dictan los criterios de lo que debe ser una investigación, generando un sesgo epistemológico y contribuyendo a la estandarización del conocimiento. Ello crea un bucle donde se valoran, se financian, se investigan y se publican sólo unas formas de conocimiento científico, que asientan tendencias y autorías dificultando el desarrollo de "emergencias o de propuestas heterodoxas" (Herrán, 2013).

Cada campo disciplinar alberga diversas concepciones respecto a qué es una investigación (fundamentos epistemológicos), cómo se debe realizar (metodologías), cómo se ejecuta (prácticas), cómo se comunica (hábitos de comunicación) y cómo se evalúa (sistemas de evaluación) (Delgado-López-Cózar, et al., 2007). Sin embargo, 
el "esquematismo científico" impulsa la escritura en ciencias humanas hacia la homogenización y estandarización (Ortiz, 2013). El campo de las Humanidades y las Ciencias Sociales, donde se ha publicado en una amplia variedad de medios y formatos, con especial predilección por las monografías, se ve ahora constreñido por un modelo estandarizado que impone como género literario el artículo (Herrán, 2013).

Ello tiene reflejo en la formación de los jóvenes investigadores, centrada en la adquisición de nuevas herramientas, aprendizajes formales y mecánicos, asociadas muchas veces a nuevas tecnologías descontextualizadas del corpus de las CC.SS. Sin embargo, la técnica no es el único constituyente de la ciencia, ni puede sustituirla, esta requiere igualmente el conocimiento de teorías y contextos, la conjunción de datos empíricos y enfoques reflexivos a partir del conocimiento disponible.

De aquí la importancia que tiene para la construcción del conocimiento la innovación metodológica, más allá la impostura de las Ciencias Técnicas, desarrollando nuevas estrategias adaptadas a la complejidad propia de las Ciencias Sociales. En el ámbito de la investigación cualitativa (ver Mannay, 2017), se sugieren nuevas formas de producción de datos mediante la incorporación de métodos como la antropología visual, la narrativa transmedia, la foto-elucidación... (Stanczak, 2007), nuevas "cartografías contrahegemónicas" y metodologías participativas que incluyan a sectores invisibilizados (Habegger y Mancila, 2005), el "arte participativo", el uso de semánticas, teorías y métodos dramatúrgicos (Morén, 2013). Y, en general, el desarrollo de estrategias a partir de conceptualizaciones previas, como los métodos autorreflexivos utilizados en la investigación (McKenney y Reeves, 2012) basados en el práctico reflexivo de Donald Schön (1987). Pero las crisis de investigación no se superan solo incorporando nuevos métodos de recogida y tratamiento de la información, son crisis del conocimiento, crisis de percepción, que requieren para su resolución nuevas epistemologías, en la línea de las aportaciones de autores como Paulo Freire, Jesús Ibáñez, Tomás Rodríguez Villasante, Boaventura De Sousa Santos, Fritjof Capra, Álex Curbero, Dolores Hernández, etc.

\subsection{Equidad y Justicia distributiva versus Desigualdad y Precariedad}

Autores como Ready (2014), han denunciado el deterioro de las condiciones de trabajo de los jóvenes investigadores en los últimos años. El incremento de los contratos temporales y el empeoramiento de las condiciones laborales atentan directamente contra la libertad académica, creando un mayor abismo entre el trabajo académico y el capital académico. Todo ello en un contexto donde el "mobbing" en el profesorado universitario va en aumento. Refiriéndose a España, Alonso Rocafort (2003) identifica esta tendencia a partir de factores como docencia con grupos masificados, burocracia asfixiante, actividad investigadora interminable, procedimientos de promoción injustos, salarios inadecuados, sistemas de apadrinamiento que generan relaciones de vasallaje, espacios de impunidad... y miedo al poder.

Por otra parte, la aparente objetividad del modelo indexado no siempre se corresponde con la realidad. Olivas-Ávila y Musi-Lechuga (2014), que analizan la vali- 
dez discriminativa e informativa del índice $\mathrm{H}$ de los miembros de la WoS de los cuerpos de profesores de Psicología en España, muestran que los sistemas integradores de productos de investigación, como el Researcher ID, contienen "imprecisiones y errores considerables, tales como duplicidad de registros y la inclusión de registros ajenos a la Web of Science. La magnitud de datos incorrectos supone graves problemas de validez y fiabilidad, que hacen que sean inadecuados para evaluar a los investigadores o para realizar cualquier tipo de estudio con los datos que contienen" (op.cit, pp. 66). Ni siquiera la revisión doble-ciega (double-blind), que descansa en el anonimato, es garantía de una selección basada en la calidad. Post et al. (2013, pp. 9, citando a Stefan Hirschauer, 2010) recuerdan que, a diferencia de los juicios silenciosos de un lector individual en la revisión por pares, en el pasado se ejercía una evaluación pública, donde el consejo editorial discutía como consejo la potencialidad y los límites de cada publicación.

Las revistas científicas funcionan como empresas, su producción está sujeta a la ley del mercado de la oferta y la demanda. En momentos de una gran oferta de artículos el nivel de exigencia tiende a aumentar, de forma que la aceptación de los artículos depende de la oferta acumulada. La ingente cantidad de producción crea una avalancha que genera un "embudo" que afecta a quien investiga y atasca la difusión de trabajos merecidos (Herrán y Villena, 2016). ¿Qué revista científica ha suspendido una edición porque los artículos disponibles no alcanzasen la calidad suficiente? La escasez de financiamiento público junto con el incremento de solicitantes han generado un sistema de cuello de botella que exige aplicar mecanismos restrictivos que limiten, "objetivamente" , el acceso a los diferentes rangos creados, los cuales quedan saturados. Esto desvela el algoritmo oculto del sistema: son muchos los llamados y pocos los elegidos.

En esta la lucha por situar artículos en las mejores revistas o por entrar en el selecto grupo de las revistas indexadas, solo una minoría lo consigue. En España, el $20 \%$ de las revistas de CC.SS. concentran el $80 \%$ de las citas. La distribución desigual de los factores que facilitan la productividad investigadora contribuye a competir en condiciones de desigualdad según disponibilidad de recursos, presencia de grupos consolidados, existencia de relaciones con empresas, etc., mayormente en momentos donde las políticas derivan hacia la financiación privada, a la que no todas las áreas tienen igual acceso.

\subsection{Sistemas Responsables de Reconocimiento versus Mercantilismo}

Un índice que se creó para evaluar las revistas y para que los bibliotecarios seleccionasen las mejores, no debe aplicarse para evaluar la ciencia. Como recuerda de la Herrán (2013), en España, el sistema de evaluación de la investigación surge con un decreto de retribuciones del profesorado que inicialmente pretendía introducir

4. La "objetividad" aquí se basa en construir una razón indiscutible para evitar el cuestionamiento de algo (reificar), en este caso, la inexistencia de recursos suficientes para financiar la carrera académica, sin discutir la racionalidad o no de la misma. 
un complemento de productividad para incentivar la actividad investigadora; pero ha derivado en un instrumento para el ascenso en la carrera profesional -acreditación- y la obtención de fondos de investigación. La actual política de reconocimiento, debido a la ambigüedad de su finalidad — incentivar la excelencia investigadora o incorporar un complemento salarial? - responde más a las necesidades de ascenso y mejora económica que a las exigencias de una investigación de relevancia y rigor. A través de una gestión empresarial-comercial, la investigación se transforma de una actividad académica y de servicio social a una función de mercado. Lo que comenzó siendo una forma de evaluación de la calidad de las publicaciones desde y para la comunidad científica ha derivado en un complejo sistema de producción regulado por un mercado, más externo que interno, que convierte a la investigación en un asunto empresarial rentable.

Proliferan las asesorías a docentes universitarios, surgen especialistas sobre cómo publicar un artículo, cómo obtener un proyecto, cómo incluir una revista en Scopus o JCR o cómo mejorar el índice H. Lo primero que aconsejan estos "especialistas", expertos en las transacciones y fórmulas que regulan el mercado del intercambio académico, no es sobre qué ni cómo investigar, sino dónde y cómo publicar. Aparecen manuales de supervivencia, editados por las propias empresas editoriales (ej: «Cómo trazar el itinerario para alcanzar el éxito como investigador profesional», Johnson, 2011; «Los diez mandamientos para incrementar las citas», Buela-Casal, 2002); se ofertan cursos para publicar en revistas de impacto, congresos que premian con la publicación de las aportaciones en revistas indexadas, programas de doctorado que exigen para defender la tesis haber publicado previamente... El manual APA (American Psychological Association) se ha convertido en el catecismo del investigador de las ciencias sociales.

La evaluación vinculada a incentivos suele conllevar un aumento de las publicaciones científicas y de la producción de nuevos conocimientos; pero no garantiza la relación de esos conocimientos con la mejora social. Es preciso un modelo de impacto que sea coherente con la función social de la universidad, no dictado por los monopolios editoriales, basado en la construcción de conocimientos orientados a la solución de problemas, en su aplicación a la práctica profesional, social y política, que llegue a los ámbitos de decisión. Una cosa es el impacto de un artículo en la academia y otra su impacto en la realidad social y en los agentes que intervienen en esa realidad. El impacto y la transferencia se ven facilitados cuando los actores se implican en las actividades del proyecto, en el proceso de co-creación de resultados (involve stakeholders).

\section{COMBATIR LA TIRANÍA DEL FACTOR DE IMPACTO}

\subsection{Establecer un nuevo sistema de evaluación de la actividad investigadora compatible con la calidad docente}

Ante la presión por la eficiencia y el instrumentalismo que ahoga la creatividad, el descubrimiento y la mejora social, es necesario plantear una evaluación diferente. Las evaluaciones aplicadas masivamente, sea en el ámbito de la investi- 
gación o de la docencia, tienden a deteriorarse; evaluar mucha investigación, muy diversa, de forma estandarizada y cuantitativa lleva a una simplificación de los procesos que desvirtúa su finalidad básica. Muchas publicaciones que se evalúan positivamente no tienen detrás el soporte en una investigación sólida. Los llamados "tramos de investigación" deberían llamarse mejor "tramos de publicación", puesto que prácticamente solo se evalúan publicaciones. "Aunque las políticas [de estímulo] buscaban instaurar una cultura de la evaluación, lo que los distintos programas de evaluación generaron fue un aparato burocrático dedicado al recuento curricular" (Buendía et al. 2017, pp. 980). Ante esta realidad, estos autores (op.cit.: 983) proponen una evaluación académica concebida como una evaluación diagnóstica, no centrada exclusivamente en los productos sino en el análisis integral de los procesos, condiciones y contextos de trabajo; y una evaluación formativa, entendida como un ejercicio de reflexión colegiada, de retroalimentación y de intercambio informado entre colegas que asumen el papel de interlocutores y no de jueces, cuyo propósito central sea la mejora de los procesos de trabajo, la superación continua de las personas, los grupos y las instituciones encargadas de llevarlos a cabo.

Manzano-Arrondo (2017, pp. 11 y 28) afirma que "los mecanismos de evaluación muestran un marcado comportamiento cíclico, tautológico y no coherente con los criterios que han caracterizado el desarrollo de la ciencia... y que la evaluación de la ciencia debería ser científica, no acomodaticia". Para ello sugiere un cambio paradigmático en la evaluación de la actividad científica, consistente en:

Utilizar de preferencia métodos cualitativos.

- Generar sistemas muy sensibles a la amplia diversidad de situaciones y contextos de investigación. Usar referentes y no ordenaciones.

- Considerar el amplio abanico de actividades investigadoras, buena parte de las cuales no se traduce en publicaciones.

- Incluir como criterio las repercusiones que la actividad científica genera fuera del ámbito expreso de la ciencia, como ocurre con el impacto social.

- Practicar una transparencia absoluta, que incluye la participación de los agentes implicados, para garantizar que el método no se anquilose.

La irrupción y el éxito del open access no supone abandonar la exigencia de calidad para la publicación científica; pero sí puede, si así lo quiere la comunidad científica, revertir el sistema establecido de evaluación basado en el empleo de una o dos fuentes de información (WoS y Scopus), con grandes solapamientos entre ambas, con fuertes intereses comerciales y con las citaciones como bastión de calidad de las publicaciones; para lo que sería conveniente contar con fuentes de indicadores independientes y de ámbito nacional, que puedan constituir un apoyo en las evaluaciones sin estar mediatizadas por grupos empresariales (Giménez Toledo, 2014). 


\section{EST UDIOS Y ENSAYOS}

A continuación, destacamos algunas medidas dirigidas a mejorar la evaluación de la investigación:

- Profundizar y compartir un concepto enriquecido de ciencia y de evaluación, con una apertura metodológica que atienda a las características de los diferentes campos científicos -también las Artes y las CC.SS.-; valorar y pretender la complementariedad metodológica; equilibrar la investigación empírica y reflexiva; evaluar trabajos con una intencionalidad didáctica; superar las barreras entre disciplinas académicas, que parcelan el conocimiento artificialmente (Herrán y Villena, 2012).

- Incluir todas las revistas y soportes, utilizar indicadores a nivel de artículo y no solo de revista. Los indicadores a nivel de artículo no solo se refieren a las citas recibidas por un trabajo en particular, sino que recaban también los que entran dentro de "Almetrics", es decir, los relacionados con las descargas del artículo, los comentarios sobre él, los tuiteos y retuiteos, los marcadores en Mendeley o Delicious, más todas las informaciones que puedan generarse en las redes sociales académicas (Giménez Toledo, 2014).

- Reforzar la evaluación de grupos y unidades organizativas, y no solo la productividad individual. Autores como Arencibia y de Moya Anegón (2008) consideran necesaria la revisión de los indicadores cienciométricos utilizados y proponen una nueva perspectiva del desarrollo científico basada en redes de colaboración.

- Dar estabilidad al modelo. Los criterios de evaluación se modifican, a veces cada año, creando gran incertidumbre. Esta variabilidad cuestiona su adecuación para ser aplicados a la actividad investigadora, que exige una continuidad a medio plazo.

- Evitar el solapamiento y redundancia de evaluaciones. Las agencias de evaluación estatales, las regionales y las propias universidades realizan evaluaciones simultáneas de la producción científica, utilizando como indicador el número de sexenios, de forma que un mismo criterio puntúa varias veces. A su vez, un mismo investigador, proyecto o equipo puede ser evaluado por varias agencias a la vez, agencias que actúan de forma independiente unas de otras, por lo que pueden darse resultados discrepantes, incluso "dobles financiaciones", a un mismo proyecto o mérito. La multiplicación de agencias de evaluación exige mejorar su coordinación, incluyendo la de sus bases de datos.

- Dar mayor transparencia a los procesos de evaluación, explicitando al máximo los criterios y procedimientos de decisión, incluido el derecho a la reclamación, sin por ello judicializar las decisiones en extremo, que beneficia a quienes poseen mayores recursos para pleitear. También debe haber transparencia en los procesos de nominación y gestión de las comisiones de evaluación, que no deberían ser de carácter voluntario. 
- Hacer metaevaluación de los efectos del modelo. Toda evaluación es mejorable técnicamente, pero el problema también recae en las decisiones políticas que se toman a partir de los resultados, que con frecuencia se utilizan mal, sesgadamente, para premiar o castigar, no para mejorar.

Las medidas anteriores, aun siendo necesarias, no garantizan un cambio sustancial en el sistema si no van acompañadas de una reorganización del trabajo académico coherente y dirigido a diversificar el modelo de carrera académica, frente al modelo único que penaliza la docencia. El descrédito de la docencia y la sobreincentivación de la investigación están llevando a una desatención de la enseñanza, considerada como una actividad secundaria. Para dotar plazas de profesorado se exigen necesidades docentes, pero para seleccionar al profesorado se valora la investigación como criterio principal. Esto está provocando el ingreso de jóvenes en la docencia universitaria con escasa competencia docente; así como la existencia de buenos docentes que, sobrecargados de docencia, se ven relegados en la promoción como penalización por no acreditar suficiente producción investigadora, lo que genera grupos de docentes objetores a la investigación. Evitar la descompensación entre la evaluación de la investigación y la evaluación de otras funciones esenciales como la docencia, la transferencia o la innovación requiere entender la evaluación de la producción investigadora como parte de una concepción del desempeño profesional más amplia, que permita contemplar el impacto de la actividad investigadora en el conjunto de funciones y tareas que realiza el profesorado universitario, considerando su trayectoria, recursos y perfil completo. Una incentivación ética requiere tener en cuenta las condiciones de producción de la investigación.

La investigación no puede ser la única vía para hacer carrera profesional universitaria. Esto provoca que la mayoría aspire a ello y genere carreras frustradas. Establecer un doble modelo de carrera universitaria, docente e investigadora, permitiría evaluar los dos perfiles con rigor, según la naturaleza de su actividad, de forma que desde ambos se pueda obtener la excelencia. La excelencia docente no garantiza la excelencia investigadora ni viceversa, ambas se deben realimentar, especialmente la docencia respecto a la investigación, aunque exijan competencias diferentes cuyo dominio es igualmente meritorio. Ello evitaría que todos quieran/deban ser investigadores, que muchos tengan que investigar en precario y que los buenos docentes se sientan discriminados respecto a los investigadores.

\subsection{Repensar las prácticas de investigación en el contexto académico}

Reconceptualizar la ética y validez de la investigación exige modificar las agendas de investigación y difusión académicas. Entre los requisitos de una investigación ética están:

- Que sea de calidad: rigurosa, fiable, creíble.

- Que tenga utilidad social, más vinculada al contexto, dirigida a la solución de problemas sociales, a mejorar la vida de las personas, con énfasis en los colectivos más necesitados. 
- Que cumpla criterios éticos en su diseño, financiación, desarrollo, gestión y utilización: transparencia, no manipulación, no dominio, no explotación de las personas, inclusividad, difusión abierta y uso social.

Autores como Gendron (2008) o Herrán y Villena (2016) señalan que el estado de cosas se sostiene a través de un sistema de complicidad institucional, según el cual las administraciones públicas (los sistemas nacionales de ciencia y tecnología), pero también las universidades, son cooperadores necesarios de la empresa correspondiente -Clarivate Analytics- para el desarrollo del sistema comercial de evaluación. En este escenario gerencialista, el desafío consiste en repensar la práctica investigadora, ensanchando el debate al sistema de valores éticos que preside la gestión de la investigación (Sánchez Blanco, 1997). ¿Es posible llevar a cabo una investigación crítica y orientada a la mejora social desde la universidad?

¿Por dónde comenzar? Marcela Mollis (2008, pp. 10), profesora de la Universidad de Buenos Aires, sugiere que "habrá que reconocerse para cambiar..." Reconocer la necesidad de reconstruir la misión institucional de la universidad, reconocer la crisis de representatividad de los cuerpos colegiados y la disfuncionalidad de la estructura administrativa, reconsiderar la relación entre ciudadanía política universitaria y selección académica por concurso en base a méritos, "pedagogizar los problemas de la universidad, politizarlos y despartidizarlos".

Ante el poder que ejercen las editoriales sobre la carrera de los investigadores, las revistas académicas y las asociaciones que las sustentan deben reaccionar modificando sus políticas editoriales, en línea de lo propuesto por Post et al. (2013, pp. 12-13), quienes sugieren varias opciones, como publicar los artículos de las revistas académicas simultáneamente en un medio abierto, resucitar la función de comunicación entre las redes académicas, crear un archivo abierto con los reportes de los evaluadores y las respuestas de los evaluadores a los autores... lo que permitiría juzgar la calidad de otras revistas. Y hacen una llamada a los consejos editoriales a tomar control de sus propios guiones con el fin de evitar, o al menos reconocer, las desventajas del sistema actual: "Esperamos que las revistas comiencen a identificar significados y medidas de calidad independientes, y que consideren la comunicación y el compromiso como elementos primordiales dentro de sus objetivos" (op. cit, pp. 13).

Frente a los excesos burocráticos de la evaluación de la ciencia, la "Declaration on Research Assessment de San Francisco" de 2012, conocida por las siglas DORA5, recomendaba actuar en tres niveles: eliminar el factor de impacto de las revistas en los procesos de contratación y promoción del personal investigador y en la financiación de la ciencia; evaluar la investigación por su mérito intrínseco y no en función de la revista en que se publica; y capitalizar las oportunidades de la publicación en

5. Varios editores y empresas editoras de revistas científicas académicas se dieron cita en San Francisco en diciembre de 2012, coincidiendo con la reunión anual de la Sociedad Americana de Biología Celular. Allí acordaron hacer una declaración conjunta que se ha dado en llamar el espíritu DORA (del inglés, “Declaration on Research Assessment”) http://am.ascb.org/dora/. 


\section{EST UDIOS Y ENSAYOS}

abierto vía internet, con menos limitaciones en el número de palabras, citas y figuras, buscando nuevos indicadores del significado e impacto de los trabajos, como el índice h de Hirsch. El "Manifiesto de Leiden" del $2015^{6}$ insiste en que la investigación para avanzar en las fronteras del conocimiento académico es diferente de la investigación centrada en proveer soluciones a problemas sociales. En España, la "Declaración del Foro de Sevilla sobre la política de sexenios y la evaluación del profesorado universitario"7 constituye un manifiesto frente al modelo actual dominante.

En el marco actual del neoliberalismo económico y político, es necesario ir más allá del ámbito universitario. "La excelencia académica tiene que ver con la democratización efectiva de nuestras universidades, con la democratización de las formas de producción y difusión de saberes socialmente significativos y con la propia democratización de las posibilidades de acceso y permanencia de los más pobres en las instituciones de educación superior" (Gentili, 2008, pp. 11).

Necesitamos nuevas narrativas que inviten a crear y difundir el conocimiento de formas alternativas. Frente a la eficiencia y el instrumentalismo metodológico, que han reducido la creatividad investigadora, y en pleno auge del productivismo -producir mucho y en poco tiempo-, es preciso reivindicar la "ciencia lenta" (slow science) (Salazar y Rivoal, 2013), la creación y transferencia basadas en las artes y en otros lenguajes comunicativos que generen flexibilidad, serendipia, potencien el valor de la representación, la interacción, la interpretación, la crítica, la confianza, el autoconocimiento.

Conviene recordar lo que a menudo se considera como la característica principal de la academia: el alma de las universidades es la libertad académica -la libertad de investigación, la libertad de enseñanza, la libertad de expresión y de disidencia, la libertad de publicar, la libertad de expresar opiniones sobre la institución en la que se trabaja-. Todas estas libertades deben ejercerse sin referencia a la ortodoncia, la sabiduría convencional o el temor a la represión del estado o de cualquier otra fuente. Sin estas libertades, las universidades no pueden cumplir su función de descubrir el conocimiento, de difundir ese conocimiento a sus estudiantes y la sociedad en general, y de inculcar a sus estudiantes una madura independencia mental (Turk, 2004, pp. 11-12, citado por Gendron, 2008, pp. 122)

Cabe preguntarse, finalmente, si el reconocimiento es una prioridad del sistema. Sobredimensionar el reconocimiento como tarea de la justicia puede llevar a desatender otros conflictos de fondo como el del acceso a los recursos escasos, la igualdad y la libertad (Grueso, 2017). En cualquier caso, las políticas del reconocimiento deben integrar las políticas de la redistribución y de la justicia social, más allá de la institución donde se aplican.

6. http://www.leidenmanifesto.org/

7. http://bit.ly/2sf5caY 


\section{ESTUDIOS Y ENSAYOS}

\section{REFERENCIAS}

Alonso Rocafort, V. (2014, 21 de noviembre). El agujero negro de la Universidad española, El Diario.es, http://bit.ly/2quVwZq

Arencibia, J. y Moya Anegón, F. de (2008). La evaluación de la investigación científica: una aproximación teórica desde la cienciometría, ACIMED, 17 (4), http://bit.ly/2QC8IMo

Buela-Casal, G. (2002). La evaluación de la investigación científica: el criterio de la opinión de la mayoría, el factor de impacto, el factor de prestigio y Los Diez Mandamientos para incrementar las citas. Análisis y Modificación de Conducta, 28 (119), 455-475, http:// bit.ly/2YE0ijZ

Buendía, A., García Salord, S., Grediaga, R., Landesman, M., Rodriguez-Gómez, R.; Rondero, N., Rueda, M. y Vera, H. (2017). Queríamos evaluar y terminamos contando. Alternativas para la evaluación del trabajo académico. Perfiles Educativos, 39 (157), 1-19. http://bit.ly/2P78aY7

Calver, M., Lilith, M. y Dickman, Ch. (2013). Un 'incentivo perverso’ de la bibliometría: ¿podrían los Ejercicios de Evaluación de Investigación Nacional (NRAE) restringir la disponibilidad de literatura para la conservación de la naturaleza? Scientometrics, Akadémiai Kiadó. Springer, 95 (1), 243-255, https://doi.org/10.1007/s11192-0120908-1

Cheek, J. (2013). La práctica y la política de la investigación cualitativa financiada. En Denzin, N.K. y Lincoln, Y. (coords.). Manual de investigación cualitativa, 3, (pp. 52-93). España: Gedisa

Delgado-López-Cózar, E., Ruiz-Pérez, R. y Jiménez-Contreras, E. (2007). La Edición de Revistas Científicas: Directrices, Criterios y Modelos de Evaluación, http://bit.ly/20uqMjs

Fang, F.; Steen, G. y Casadevall, A. (2012). Misconduct accounts for the majority of retracted scientific publications. PNAS, Edited by Thomas Shenk, Princeton University, 109 (42). http://10.1073/pnas.1212247109

Fernández-Riosa, L. y Rodríguez-Díaz, J. (2014). The 'impact factor style of thinking': A new theoretical framework. International Journal of Clinical and Health Psychology, 14, 154-160, https://doi.org/10.1016/s1697-2600(14)70049-3

Gendron, Y. (2008). Constituting the Academic Performer: The Spectre of Superficiality and Stagnation. Academia European Accounting Review, 17(1), 97-127, https://doi. org/10.1080/09638180701705973

Gentili, P. (2008). Posneoliberalismo, Reforma Universitaria y Excelencia Académica. Cuadernos del Pensamiento Crítico Latinoamericano. Buenos Aires: Consejo Latinoamericano de Ciencias Sociales, CLACSO, n‥ 13, octubre, 2008, http://bit.ly/2KH4w4D

Gibbons, M., Limoges, C., Nowotny, H., Schwartzman, S., Scott, P. y Trow, M. (1997). La nueva producción del conocimiento. La dinámica de la ciencia y la investigación de lassociedades contemporáneas. Barcelona: Pomares-Corredor https://doi.org/10.2307/2076669

Gibert-Galassi, J. (2013). Autonomía y dependencia en las ciencias sociales latinoamericanas. Un estudio de bibliometría, epistemología y política. Buenos Aires: CLACSO, http://bit. ly/2QCVZDu

Gibert-Galassi, J. (2015). La producción 'indexada' en las ciencias sociales latinoamericanas: 1993-2012". Revista Latinoamericana de Investigación Crítica, II (2), 19-40. http://bit.ly/2qHMSqO

Giménez Toledo, E. (2014). Imposturas en el ecosistema de la publicación científica. Revista de Investigación Educativa, 32 (1), 13-23, http://dx.doi.org/10.6018/ rie.32.1.190251 


\section{ESTUDIOS Y ENSAYOS}

Grueso, D. (2017). Injusticia y Luchas por el Reconocimiento. ¿Objetividad Cognitiva o Preeminencia Política?. Cuadernos del Pensamiento Crítico Latinoamericano, no. 42. CLACSO, http://bit.ly/344PGMV

Habegger, S. y Mancila, I. (2005). La cartografía social como estrategia para diagnosticar nuestro territorio. En Celada, B. y Habegger, S. (coords.), Interpretando a Freire. Haciendo camino desde la colectividad. Seminario de Paulo Freire. Málaga, Asociación de reflexión e innovación educativa (pp. 29-36). Colectivo Sur Ediciones, http://bit. ly/2Xvr51k

Herrán, A. De la (2013). ¿Una transdisciplinariedad inmadura? Consideraciones críticas radicales (pensando desde la Pedagogía y la Didáctica). En Paredes, J., Hernández, F. y Correa, J.M. (eds.), La relación pedagógica en la universidad, lo transdisciplinar y los estudiantes. Desdibujando fronteras, buscando puntos de encuentro (pp. 30-51). Madrid: Universidad Autónoma de Madrid, http://hdl.handle.net/10486/13152.

Herrán, A. De la y Villena, J.L. (2012). La 'cultura del impacto JCR': El caso del área de Didáctica y Organización Escolar. En Herrán, A. de la y Paredes, J. (coords.). Promover el cambio pedagógico en la universidad (pp. 287-304). Madrid: Pirámide, http://bit. ly/2QFFZ3J

Herrán, A. De la y Villena, J.L. (2016). ¿Es útil para el área de Didáctica y Organización Escolar el sistema de evaluación de la productividad científica centrado en el factor de impacto? Revista Electrónica Interuniversitaria de Formación del Profesorado, 19 (1), 215-227, http://bit.ly/34dw7S8

Jhonson, A. (2011). Cómo trazar el itinerario para alcanzar el éxito como investigador profesional, Ámsterdam: Elsevier http://bit.ly/2E1Trra

Mannay, D. (2017). Métodos visuales, narrativos y creativos en investigación cualitativa. Madrid: Narcea.

Manzano-Arrondo, V. (2017). Hacia un cambio paradigmático para la evaluación de la actividad científica en la Educación Superior. Revista de la Educación Superior, 46 (183), 1-35, http://bit.ly/2D3T3bf

Mckenney, S. y Reeves, Th. (2012). Conducting educational design research. London: Routledge. http://bit.ly/2Pa9yt7

Moed, H. F. (2005). Citation analysis in research evaluation. Nueva York: Springer. https:// doi.org/10.1007/1-4020-3714-7

Mollis, M. (2008). Recrear la comunidad académica universitaria, un imperativo necesario. Cuadernos del Pensamiento Crítico Latinoamericano, nº.13 (octubre). Buenos Aires: CLACSO, http://bit.ly/2KH4w4D

Morén, S. (2013). SoundImages. Artistic Research in Teachers Education. En F. Hernandez (ed), 1st Conference on Arts-Based and Artistic Research (157-161). University of Barcelona, Faculty of Fine Arts, http://bit.ly/2qCgqpB

Olivas-Ávila, J. A. y Musi-Lechuga, B. (2014). Validez y fiabilidad del Researcher ID y de «Web of Science Production of Spanish Psychology». International Journal of Clinical and Health Psychology, 14 (1), 58-66, https://doi.org/10.1016/s16972600(14)70037-7

Opazo, T. (2017). La tiranía de las publicaciones académicas, http://bit.ly/37tcZCv

Ortiz Naranjo, Ma . N. (2013). El latido del texto. Juegos de saber, poder y resistencia en la escritura académica en ciencias humanas. Revista Latinoamericana de Investigación Crítica, CLACSO, 2, 137-156. http://bit.ly/2sbKtoA

Palomero Pescador, J. E. (2010). Los índices de impacto, una mirada crítica. Revista Interuniversitaria de Formación del Profesorado (RIFOP), Octubre, http://bit.ly/37kFcLr 


\section{EST UDIOS Y ENSAYOS}

Post, D., Stambach, A., Ginsburg, M., Hannum, E., Benavot, A. y Bjork, Ch. (2013). Los rankings académicos. Archivos Analíticos de Políticas Educativas, 21 (19), https://doi. org/10.14507/epaa.v21n19.2013

Ready, D. (2014). From academic freedom to academic capitalism. Discover Society, February, http://bit.ly/2099RnD

Rincón Ramírez, C. (2017). Los Patriarcas del Conocimiento. La Furtividad del Poder Intelectual. Estructuras de poder en la producción del conocimiento. México: Universidad Autónoma del Estado de Chiapas (mimeo).

Salazar, N. y Rivoal, I. (2013). Contemporary Ethnographic Practice and the value of serendipity. Social Antropology, 21 (2), 178-185, https://doi.org/10.1111/1469-8676.12026

Sánchez Blanco, C. (1997). Dilemas éticos de la investigación educativa. Revista de Educación, 312, 271-280. http://bit.ly/2RDxAOF

Santos Rego, M. Á. y Fernández-Ríos, L. (2016). El factor de impacto y el futuro de las revistas académicas. El riesgo de patologización. Innovación Educativa, 16 (72), 35-52, http://bit.ly/332vKcb

Sañudo Guerra, L. (2017). La generación de la cultura científica educativa en los posgrados en educación en México. Un modelo de gestión de conocimiento. En Transformative and Inclusive Social and Educational Research, 3rd International Summer Workshop on Alternative Methods in Social Research, Málaga, julio. http://bit.ly/20uLFe7

Saura, G. y Bolívar, A. (2019). Sujeto Académico Neoliberal: Cuantificado, Digitalizado y Bibliometrificado. REICE. Revista Iberoamericana sobre Calidad, Eficacia y Cambio en Educación, 17 (4), 9-26, https://doi.org/10.15366/reice2019.17.4.001

Schön, D. (1987). Educating the Reflective Practitioner. San Francisco: Jossey-Bass

Stanczak, G. C. (2007) (Ed.). Visual Research Methods. Los Ángeles, CA: Sage. https://dx.doi. org/10.4135/9781412986502

Torres López, J. (2013). Austeridad y control político del conocimiento. Rebelión. 25 de junio, http://bit.ly/203UyfK

Torrez, Y. F. (2013). Los avatares de las Ciencias Sociales. Juegos de poder y estructura académica en las carreras de sociología de las universidades públicas en Bolivia. Revista Latinoamericana de Investigación Crítica, 2, 89-110, http://bit.ly/2RFUwwH

Van Raan, A. F. J. (2005). Fatal attraction: Conceptual and methodological problems in the ranking of universities by bibliometric methods. Scientometrics, 62 (1), 133-143, https://doi.org/10.1007/s11192-005-0008-6

Walker, V. S. (2017). La evaluación como mecanismo de regulación del trabajo académico. Estudio de casos en universidades de Argentina y España. Archivos Analíticos de Políticas Educativas, 25 (108), http://dx.doi.org/10.14507/epaa.25.2681

Wouters, P. (1999). The citation culture. Tesis doctoral. Amsterdam, University of Amsterdam. http://bit.ly/2Yy4UrQ 